\title{
Proton acceleration by irradiation of isolated spheres with an intense laser pulse
}

\author{
T. M. Ostermayr,,${ }^{1,2,{ }^{*}}$ D. Haffa, ${ }^{1}$ P. Hilz, ${ }^{1}$ V. Pauw,${ }^{3}$ K. Allinger, ${ }^{1}$ K.-U. Bamberg, ${ }^{3}$ P. Böhl,,${ }^{3}$ C. Bömer, ${ }^{1}$ P. R. Bolton, ${ }^{1}$ \\ F. Deutschmann, ${ }^{3}$ T. Ditmire, ${ }^{4}$ M. E. Donovan, ${ }^{4}$ G. Dyer, ${ }^{4}$ E. Gaul,${ }^{4}$ J. Gordon, ${ }^{4}$ B. M. Hegelich,,${ }^{4}$ D. Kiefer, ${ }^{1}$ C. Klier, ${ }^{3}$ \\ C. Kreuzer, ${ }^{1}$ M. Martinez, ${ }^{4}$ E. McCary, ${ }^{4}$ A. R. Meadows, ${ }^{4}$ N. Moschüring, ${ }^{3}$ T. Rösch, ${ }^{1}$ H. Ruhl, ${ }^{3}$ M. Spinks, ${ }^{4}$ \\ C. Wagner, ${ }^{4}$ and J. Schreiber ${ }^{1,2}$ \\ ${ }^{1}$ Ludwig-Maximilians-Universität München, Am Coulombwall 1, 85748 Garching, Germany \\ ${ }^{2}$ Max-Planck-Institut für Quantenoptik, Hans-Kopfermann-Str. 1, 85748 Garching, Germany \\ ${ }^{3}$ Ludwig-Maximilians-Universität München, Theresienstr. 37, 80333 München, Germany \\ ${ }^{4}$ Center for High Energy Density Science, C1510, University of Texas at Austin, Austin, Texas 78712, USA
}

(Received 18 April 2016; published 26 September 2016)

\begin{abstract}
We report on experiments irradiating isolated plastic spheres with a peak laser intensity of $2-3 \times 10^{20} \mathrm{~W} \mathrm{~cm}^{-2}$. With a laser focal spot size of $10 \mu \mathrm{m}$ full width half maximum (FWHM) the sphere diameter was varied between $520 \mathrm{~nm}$ and $19.3 \mu \mathrm{m}$. Maximum proton energies of $\sim 25 \mathrm{MeV}$ are achieved for targets matching the focal spot size of $10 \mu \mathrm{m}$ in diameter or being slightly smaller. For smaller spheres the kinetic energy distributions of protons become nonmonotonic, indicating a change in the accelerating mechanism from ambipolar expansion towards a regime dominated by effects caused by Coulomb repulsion of ions. The energy conversion efficiency from laser energy to proton kinetic energy is optimized when the target diameter matches the laser focal spot size with efficiencies reaching the percent level. The change of proton acceleration efficiency with target size can be attributed to the reduced cross-sectional overlap of subfocus targets with the laser. Reported experimental observations are in line with 3D3V particle in cell simulations. They make use of well-defined targets and point out pathways for future applications and experiments.
\end{abstract}

DOI: 10.1103/PhysRevE.94.033208

\section{INTRODUCTION}

Highly localized plasmas produced by intense laser pulses played an important role in the early development and understanding of laser plasma physics [1-4]. Meanwhile, the $10 \mathrm{GW}$ giant laser pulses from the 1960s constitute the pedestal or prepulse of today's super-intense chirped pulse amplified Petawatt lasers. Theoretical studies at presently achieved laser intensities have elucidated scenarios that make use of isolated mass-limited targets often showing enhanced ion energies and peaked spectral distributions which may be favorable for applications [5-11]. Recent experimental approaches to realize mass-limited targets include water droplets with diameters typically in the $10 \mu \mathrm{m}$ range [12-14], gas-cluster targets in the $100 \mathrm{~nm}$ range [15-17], and objects held by thin structures in the $100 \mathrm{~nm}$ to few $\mu \mathrm{m}$ range $[18,19]$. In all these cases the range of accessible target diameters is limited, while the next solid structure is either directly connected or near the target at distances of few micrometers and/or the target is surrounded by significant amounts of gas. Such nearby structures (e.g., neighboring targets) have been shown to contribute to the target interaction [15,20]. In this paper we present the first experiment using truly isolated and well-defined polystyrene (C8H8) spheres as targets, spanning an unprecedented range of target diameters. Using such targets with high-energy laser pulses enables the experimental test of several theoretically

\footnotetext{
*tobias.ostermayr@physik.lmu.de
}

Published by the American Physical Society under the terms of the Creative Commons Attribution 3.0 License. Further distribution of this work must maintain attribution to the author(s) and the published article's title, journal citation, and DOI. suggested regimes. Generated particle beams will facilitate applications that rely on the limited number of target particles and on the inherently small source size in a hitherto unavailable particle energy range from such targets.

\section{EXPERIMENTAL SETUP}

A schematic of the experimental setup is depicted in Fig. 1. We employ a Paul trap [21], which was developed particularly for the use with modern high-power laser systems to position isolated targets in the laser focus. To have a tightly focused laser pulse interacting with a micron-scale target the relative positioning must be accurate. The current Paul trap improves recent Paul trap-based laser-plasma experiments [20] regarding several key issues, thus allowing for significantly higher laser energies and a much larger range in target diameters. First, the setup employs an ion gun [22] for charging target particles instead of friction with surrounding gas. The thereby achieved orders of magnitude higher charge to mass ratios enable a reduction of the residual motion of trapped targets at similar kinetic energy. Second, the (oscillating) trajectory of a levitating target particle in the trap potential is damped by a continuously working active opto-electronic feedback loop instead of friction with buffer gas. This allows for pressures lower than $10^{-5}$ mbar during an experiment, which is a typical value for laser plasma experiments and ensures that particle diagnostics are not affected by the gaseous environment. The accuracy of target positioning is in the micron range. The solid structures closest to the target are trap electrodes at a distance of more than $5 \mathrm{~mm}$. Commercial targets [23] with diameters of $520 \mathrm{~nm}, 1.96 \mu \mathrm{m}, 4 \mu \mathrm{m}, 7 \mu \mathrm{m}$, $10 \mu \mathrm{m}$, and $19 \mu \mathrm{m}$ were irradiated, spanning more than four orders of magnitude in target mass and electron or ion number. Target diameters have typical deviations of only $2 \% \mathrm{rms}$. 


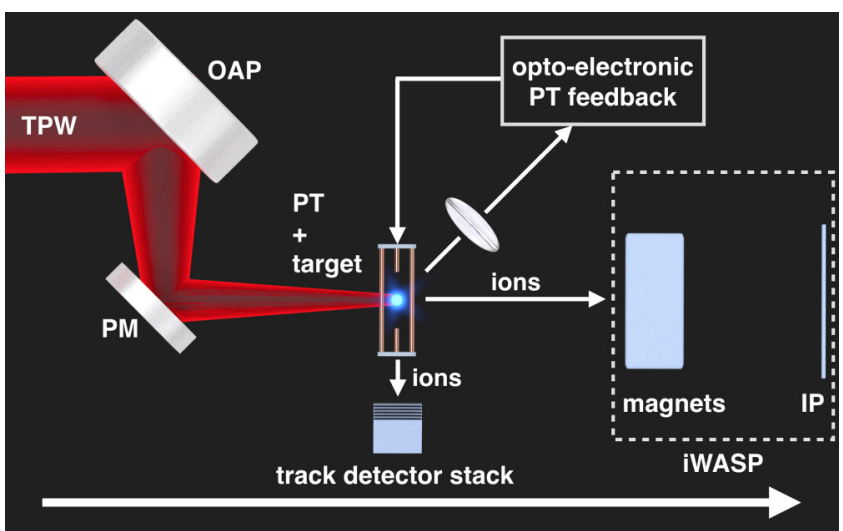

FIG. 1. Schematic of the experimental setup. All depicted components are contained in one experimental vacuum chamber. OAP stands for off axis parabolic mirror, PM stands for plasma mirror, and PT stands for Paul trap. More details regarding the setup are specified in the text.

The Texas Petawatt laser (TPW) delivered $65 \mathrm{~J}$ before the plasma mirror and $55 \mathrm{~J}$ on target in $170 \mathrm{fs}$ with a central wavelength of $1057 \mathrm{~nm}$. The laser pulse was focused by an $\mathrm{f} / 3$ off-axis parabola onto a single inline plasma mirror and subsequently to a focal spot size of $\sim 10 \mu \mathrm{m}$ FWHM and a peak intensity of $2-3 \times 10^{20} \mathrm{~W} \mathrm{~cm}^{-2}$ on target. The resulting temporal intensity contrast of the laser pulse up to several tens of ps before the peak of the main pulse was estimated of order $10^{-9}-10^{-10}$. The laser-plasma interaction was mainly characterized by the emitted energetic ions. For this purpose, nuclear track detector stacks with angular acceptance of $20 \mu \mathrm{s}$ recorded protons at kinetic energies of 3.3, 11, 17.5, 21, 26, and $28.5 \mathrm{MeV}$ emitted orthogonal to the laser propagation direction at $45^{\circ}$ with respect to the linear laser polarization. For practical reasons we accumulated shots on targets with identical diameter. Downstream of the target a wide-angle ion spectrometer (iWASP; cf. Ref. [24]) with an entrance slit perpendicular to the laser polarization and deflection magnets was used to measure angular dependent ion kinetic energy spectra for each laser shot. In the detection plane a Fuji Film image plate detector of type BAS-TR (IP) was used to detect the signal. The IP was covered by $100 \mu \mathrm{m}$ aluminum and partly covered by $1 \mathrm{~mm}$ thick CR39 nuclear track detectors in order to calibrate particle number and to distinguish carbon ions from protons via penetration depth. We did not observe a notable angular dependence of the ion signal over the accessible range of $\pm 5^{\circ}$. To isolate proton spectra in the central region we acquired protons integrated over limited angles $\left(<1^{\circ}\right)$. The $\mathrm{x}$ ray-dominated background signal plus the detector noise level have been estimated conservatively and subtracted from the spectrum for each shot. Overall 19 out of 33 shots produced a signal that can be evaluated and have been used in this analysis.

\section{RESULTS AND DISCUSSION}

Figure 2 shows the best and second best proton spectra, i.e., with the highest kinetic energies, obtained for different sphere sizes. For large spheres (19 to $7 \mu \mathrm{m}$ ) the proton differential spectra, $\mathrm{d}^{2} N / \mathrm{d} E \mathrm{~d} \Omega$, decay exponentially with kinetic energy

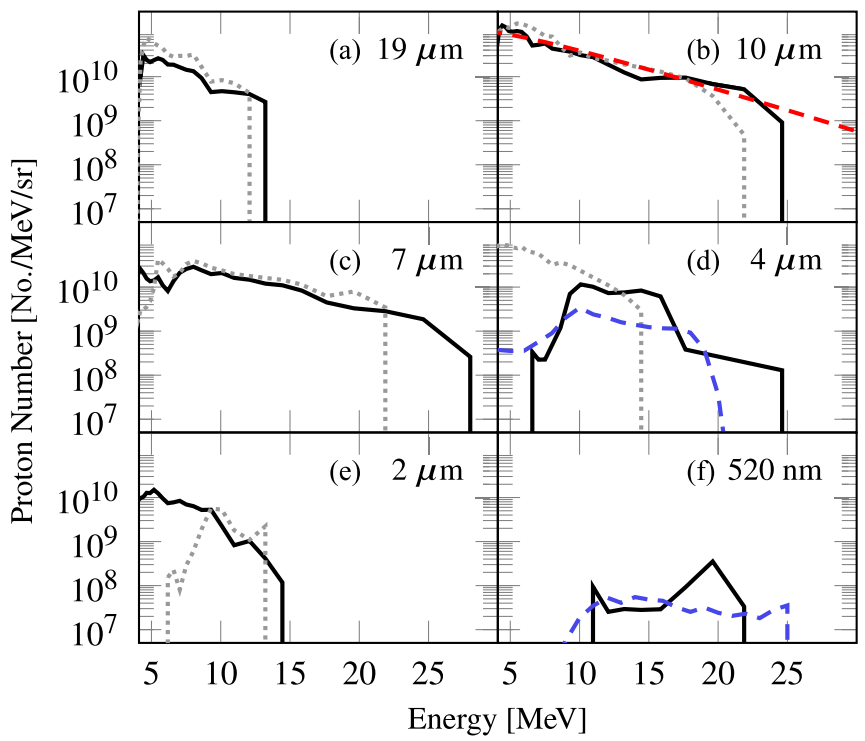

FIG. 2. Experimental proton spectra recorded with the iWASP: (a-f) Black solid lines are the proton spectra of best shots (max. kinetic energy) for the respective target size taken from the central region of the iWASP. Gray dotted lines are respective second best shots. The red dashed line in (a) is the analytical spectrum for the $10 \mu$ m case predicted [25,26] at characteristic energy of 4.2 MeV [27]. Blue dashed lines in (d) and (f) represent results of 3D particle in cell simulations described in Sec. IV $\left(d_{L}=8.3 \mu \mathrm{m}\right)$, scaled to match the respective number of target particles.

$E$, as expected from an ambipolar plasma expansion. The data are well described by the self-similar isothermal expansion model given in Ref. [25] for the spherical case or the quasineutral approach given in Ref. [26], both yielding spectral shapes of $\mathrm{d} N / \mathrm{d} E \propto\left(E / E_{0}\right)^{1 / 2} \exp \left(-E / E_{0}\right)$. Equating the characteristic (average) ion kinetic energy $E_{0}$ with the hot electron temperature of $4.2 \mathrm{MeV}$ obtained from the ponderomotive scaling [27] for our intensity, we find excellent agreement of the spectral shape, represented by the red line in Fig. 2(b) for the $10 \mu \mathrm{m}$ sphere. More interestingly, the observed maximum proton (cutoff) kinetic energy of $\sim 25 \mathrm{MeV}$ can be estimated from the same model by assuming a hot electron density of $3.4 \times 10^{23} \mathrm{~cm}^{-3}$, which is consistent with a fully ionized polystyrene sphere. This agreement is surprising since the employed ponderomotive scaling is known to overestimate the electron temperature [28] for high intensities in extended targets. Yet the ponderomotive scaling for electron temperatures has been shown via simulations to be applicable for mass-limited targets that are comparable in size to the laser focal spot size [7].

For the large $19 \mu \mathrm{m}$ spheres, both maximum proton kinetic energy and particle number are significantly reduced. This can be understood intuitively because the sphere can be regarded as a large cooling volume which dissipates energy and reduces the energy density. For slightly subfocus-sized 7 to $4 \mu \mathrm{m}$ spheres we find maximum proton kinetic energies of $28 \mathrm{MeV}$. Occasionally we observe proton kinetic energy distributions that exhibit a low-energy cutoff (i.e., nonthermal spectra). Reducing the target diameter further to $2 \mu \mathrm{m}$, the proton kinetic energies decrease while the spectral shapes remain similar to 
the $4 \mu \mathrm{m}$ case. For one shot on the smallest target used in this experiment, $520 \mathrm{~nm}$, protons are exclusively accelerated to kinetic energies between 11 and $22 \mathrm{MeV}$ while the low-energy detection limit of the spectrometer is at $3.3 \mathrm{MeV}$.

The most apparent feature in Fig. 2 is that for single shots the spectrum becomes nonthermal for sphere diameters smaller than $7 \mu \mathrm{m}$, and that maximum proton kinetic energies are reduced. We attribute this effect to a breakdown of simple (ambipolar) plasma expansion into a vacuum, which can be supported by the following estimate. Assuming a fraction $\eta_{L}$ of the laser energy $E_{L}$ is converted into hot electrons with mean energy $k_{B} T_{e}$, the number of hot electrons is of order $N_{h} \approx \eta_{L} E_{L} / k_{B} T_{e}$. For extended targets (foils or large spheres) and for the employed laser parameters and target material the absorption is likely to be $\eta_{L} \approx 0.5$ [29] and $N_{h}$ is typically much less than the number of electrons initially contained in the target $N_{T}$. For subfocus sized spheres the cross-sectional overlap of the target with the laser decreases quadratically with the target diameter, leading to a similarly decreased coupling efficiency $\eta_{L}$ of the laser. Meanwhile, $N_{T}$ decreases cubically with the target diameter. Hence, although the overall efficiency of hot electron generation is decreasing with decreased sphere diameter, $N_{T}$ is decreasing faster. A crossover (i.e., $N_{h} \approx N_{T}$ ) exists at a target diameter near $2 \mu \mathrm{m}$ for our parameter setting. For further reduced target diameters the simple estimate for $N_{h}$ is no longer applicable. In other words, smaller spheres are short on electrons to drive an efficient ambipolar expansion. Instead, the hot-electron escape depletes electrons from the sphere volume, and the ion spectra start to evince Coulomb-explosion characteristics in contrast to ambipolar expansion. For adequately small spheres the Coulomb repulsion dominates the ion acceleration. Notably, effects caused by Coulomb repulsion in multispecies targets can be observed at laser intensities much lower than the classical limit for a pure Coulomb explosion regime [30].

In fact, the absence of protons above kinetic energies of $22 \mathrm{MeV}$ in case of the $520 \mathrm{~nm}$ target allowed the distinctive measurement of maximum carbon kinetic energy for this shot, which is in the range of 104-121 MeV. This means that the slowest protons are at least as fast as the fastest carbon ions, indicating the separation of both ion species and the onset of an additional acceleration mechanism. That is driven by the Coulomb repulsion of carbon ions boosting the kinetic energy of initially slow protons inside the carbon-rich volume, thereby eliminating the low-kinetic-energy part of the proton spectrum and causing a shocklike [31] behavior as the inner protons catch up and overtake protons that were initially further out.

\section{SIMULATIONS}

In order to gain further insight to the three-dimensional dynamics, 3D3V particle-in-cell (PIC) simulations were carried out for fully ionized $500 \mathrm{~nm}, 2 \mu \mathrm{m}$, and $4 \mu \mathrm{m}$ diameter spheres using the plasma simulation code [32]. The simulated targets consist to equal parts of $C^{6+}$ and protons at an electron density of $1.7 \times 10^{23} \mathrm{~cm}^{-3}$, which corresponds to half solid density of fully ionized polystyrene. The simulation box has $2688 \times 1536 \times 1536$ grid cells with open boundary conditions at a resolution of $16 \mathrm{~nm}$ and $10^{4}$ particles per quasiparticle.
The simulated laser is a spatially and temporally Gaussian laser pulse with $55 \mathrm{fs}$ FWHM duration and $d_{L}=8.3 \mu \mathrm{m}$ or $3 \mu \mathrm{m}$ FWHM focus at a peak normalized amplitude of $a_{0}=12$. The parameters were chosen taking into account computational limitations, while keeping the $N_{h} / N_{T}$ ratio close to the experimental parameters for the respective target diameters. The simulations largely confirm the above qualitative ideas of the transition from ambipolar expansion to a Coulombrepulsion-dominated scheme by the depletion of electrons from the isolated plasma. This includes the change in the spectral distribution of protons as depicted in Figs. 2(d) and 2(f). Emulating the experimental situation, only protons emitted in a $20^{\circ}$ cone towards laser propagation direction have been taken into account for this comparison. In Fig. 3(a) it is well visible that the $4 \mu \mathrm{m}$ diameter target stays opaque during the interaction. The position of the critical density surface stays close to the initial target-vacuum interface, while short bunches of electrons are carried away in the laser propagation direction by the ponderomotive force of the laser. At the end of the simulation (231 fs after the peak of the interaction), only $0.5 \%$ of the target electrons have been removed from the vicinity of the target. The consequence is a predominant ambipolar expansion observed in the ion behavior that leads to the corresponding decaying proton kinetic energy distribution. In comparison, Fig. 3(b) shows the same laser pulse interacting with a $500 \mathrm{~nm}$ diameter target. In a similar fashion electron bunches are removed from the target that starts to disintegrate during the process. A much larger fraction of $87 \%$ of the target electrons is removed until the end of the simulation, leading to the increased impact of Coulomb repulsion between ions. Indeed, the large charge carried by the heavier and slower carbon ions pushes protons out of the carbon-rich volume, thus forming a dense expanding proton shell with a prevalence towards the target rear side.

\section{ENERGY EFFICIENCY}

We further quantify the efficiency of laser energy conversion to proton kinetic energy and compare our experimental results quantitatively to both simulations and simple estimates described above. To extract the emission angle from our experimental data we evaluate the nuclear track detector stack at $90^{\circ}$. Except for the largest sphere, the detector stacks show smaller proton numbers and energies than observed in forward direction. From that we estimate a (very coarse) opening or divergence angle of the protons between $\pi$ and $4 \pi$ sr, which agrees with simulations. Integration of the proton kinetic energy distributions in a forward direction shown in Fig. 2 over energies beyond $3.3 \mathrm{MeV}$ (iWASP detection limit) and multiplying it with $\pi$ sr, a conservative estimate for the solid angle of emission, yields the number of protons emitted from the sphere. Interestingly, for the small spheres with diameters smaller than $4 \mu \mathrm{m}$ this number approaches the number of protons initially contained in the polystyrene sphere. Furthermore we divide the kinetic energy contained in registered protons by the incident laser energy. The such defined energy conversion efficiency $\eta_{l p}$ is depicted in Fig. 4 and reaches remarkably large values of $4 \%$ for a target diameter of $10 \mu \mathrm{m}$, matching the focal spot size. As discussed before, we 

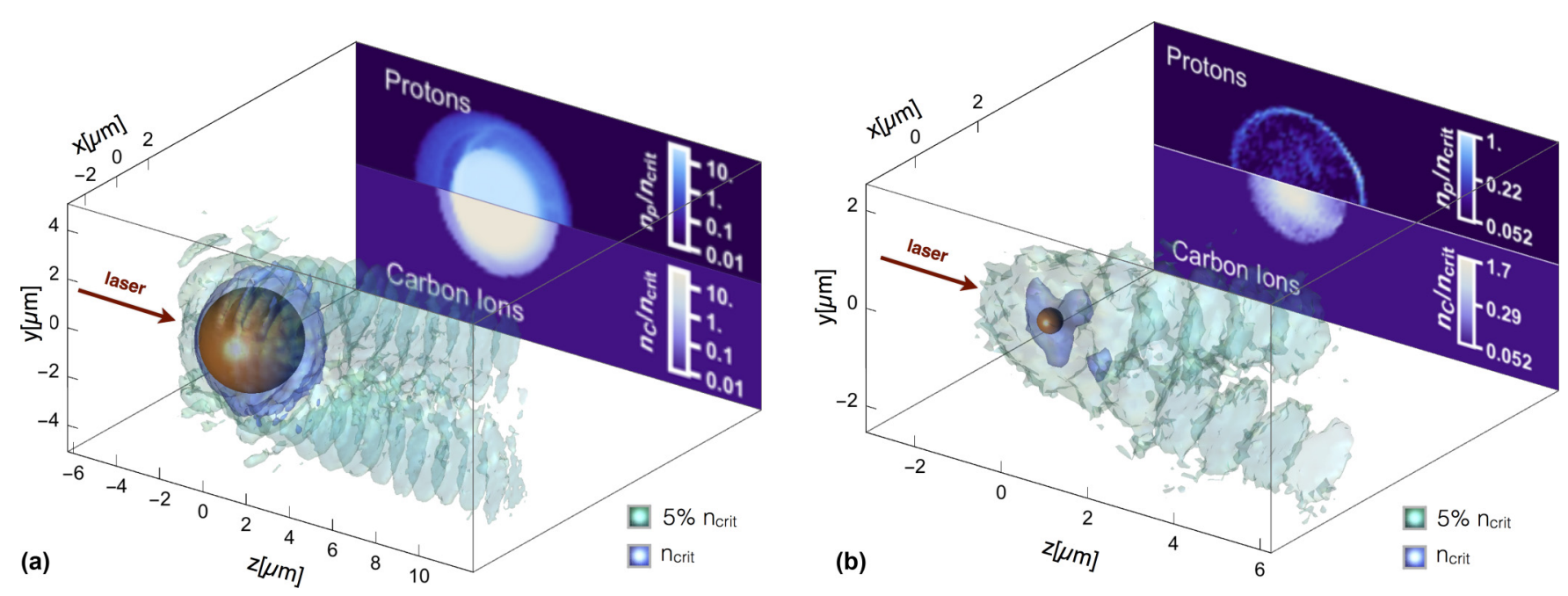

FIG. 3. Zoomed snapshots of the PIC simulation at $15 \mathrm{fs}$ after the peak interaction for (a) a $4 \mu$ m diameter target and (b) a $500 \mathrm{~nm}$ diameter target. The laser pulse is polarized in the $x=0$ plane with a peak normalized amplitude of $a_{0}=12$ and FWHM spot size of $d_{L}=8.3 \mu \mathrm{m}$. The initial target diameters and positions are indicated by the solid orange sphere at $x=y=z=0$. Electron iso-density surfaces are shown at $n_{e}=n_{\text {crit }}$ and $n_{e}=0.05 n_{\text {crit }}$, where $n_{\text {crit }}$ denotes the critical plasma density at which laser and plasma electron frequency are equal. The projection shows the proton $(y>0)$ and carbon $(y<0)$ density distribution for a $150 \mathrm{~nm}$ thick cut slice through the $x=0$ plane.

attribute the reduced laser to proton kinetic energy conversion efficiency for decreasing target diameters to the geometrically reduced cross-sectional overlap with the laser pulse. We quantify this approach assuming a Gaussian laser spot. The conversion efficiency as a function of sphere diameter is then given as $\eta_{l p}\left(d_{S}\right)=\eta_{0} \Phi\left(d_{S}\right) / \Phi(10 \mu \mathrm{m})$, where $\Phi\left(d_{S}\right)$ is the laser energy incident on the sphere with diameter $d_{S}$ and $\eta_{0}=4 \%$ as observed experimentally. For the best shots, i.e., highest maximum proton energies, the experimental conversion efficiencies (solid squares) agree well with this simple scaling. For spheres exceeding the focal spot size, the scaling predicts saturating efficiencies comparable to the TNSA case for foils. The slightly reduced values for the

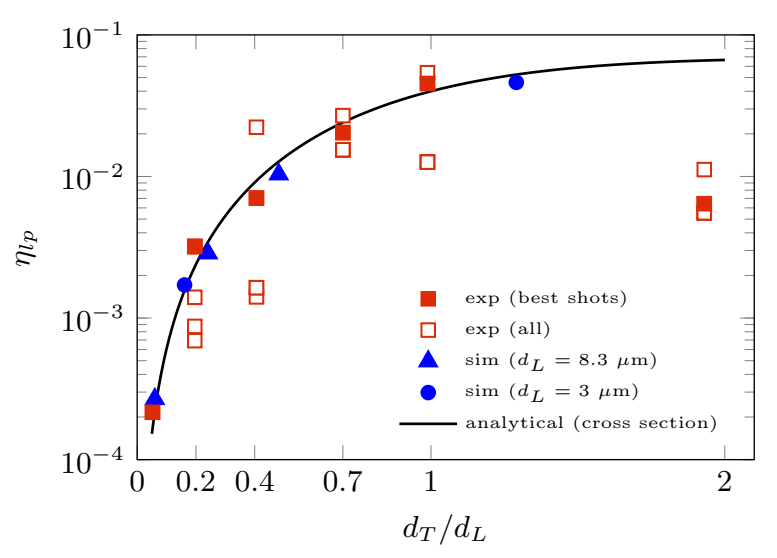

FIG. 4. Laser to proton kinetic energy conversion efficiency $\eta_{l p}$ plotted against target diameter $d_{T}$ normalized to the laser FWHM focal spot size $d_{L}$. Red squares are the experimental laser to proton kinetic energy conversion efficiencies. Solid red squares correspond to energetically best shots (solid black lines in Fig. 2). Blue triangles and circles are results of 3D3V particle in cell simulations described in the text. The black curve represents the analytical scaling taking into account the cross-sectional overlap of the target with the laser.
19.3 $\mu \mathrm{m}$ target must be treated with caution, given our strongly simplified assumption of a constant emission angle of $\pi$ sr for the differential spectra. Overall the curve shows remarkable similarities to predictions by Ref. [8] and represents the first experimental confirmation of this scenario. In the range covered by our simulations the energy conversion efficiencies observed in the simulations agree with experiments and the simple analytical scaling. Simulations performed with a smaller $3 \mu \mathrm{m}$ FWHM focal spot size indicate that indeed the reduced cross-sectional overlap with the laser rather than just the reduced surface of the target is accountable for the reduced efficiency. Hence, highly efficient proton acceleration is feasible with laser focus and target matched at smaller sizes, relaxing the currently costly requirement of large laser energy.

\section{CONCLUSIONS}

In summary, the presented study constitutes the first experimental study confirming the dependency of ion acceleration on the ratio of target diameter to laser spot size, which so far has only been treated theoretically $[7,8,33]$. We consider our results to be of general relevance for laser-driven ion accelerators. First, both the high-energy conversion efficiency in the percent range and the high maximum proton kinetic energy of $28 \mathrm{MeV}$ demonstrate the scalability of concepts tested with mass limited targets at smaller scale laser facilities (1 J level) [12,34] to higher energy lasers and consequently higher proton kinetic energies. Moreover, by varying the target diameter in an unprecedented range the results establish a qualitative idea of the laser plasma interaction with a completely isolated mass limited target. This may in combination with our detailed simulations serve as a reference for future simulations and experimental campaigns aiming to pick one single (or a more limited) set 
of target and laser parameters to focus on the understanding and optimization of one particular acceleration mechanism.

While the presented ion source does not set new world records for maximum proton kinetic energy or energy conversion efficiency from laser to ion kinetic energy from a laser-driven source, we emphasize that the well-defined proton beam from a source with real submicron source size and a well-known particle number enables unique applications that require particle energies of more than $10 \mathrm{MeV}$ from such targets. This includes special implementations of highresolution ultrafast proton radiographies, laser absorption measurements at high intensities via particle spectroscopy of all particles contained in a target, and laboratory-scale models for astrophysical processes including shock physics. Therefore our efforts extend the tradition of isolated targets for laser plasma interactions and demonstrate their potential for research and applications using cutting-edge laser technology.

\section{ACKNOWLEDGMENTS}

This work was supported by the DFG via the Cluster of Excellence Munich-Centre for Advanced Photonics (MAP) and Transregio SFB TR18. The Texas Petawatt Laser and University of Texas researchers are supported by the NNSA under Cooperative Agreement DE-NA0002008. The authors acknowledge computational resources provided by SuperMUC under Grant No. pr48me. T.M.O. and P.H. acknowledge support of Ivo Cermak and CGC Instruments in design and realization of the Paul trap system. T.M.O. and K.-U.B. acknowledge support from IMPRS-APS. D.K. acknowledges financial support from the LMUexcellent Junior Research Fund. P.R.B. and C.B. acknowledge support by DAAD. This work has been carried out within the framework of the EUROfusion Consortium and has received funding, through the ToIFE, from the European Union's Horizon 2020 research and innovation programme under grant agreement number 633053.
[1] J. M. Dawson, Phys. Fluids 7, 981 (1964).

[2] A. F. Haught and D. H. Polk, Phys. Fluids 9, 2047 (1966).

[3] T. Jarboe, Study of an isolated, laser-produced deuterium plasma in a magnetic field, dissertation, Lawrence Berkeley Laboratory and UC Berkeley (1974).

[4] H. Baumhacker, H. Brinkschulte, R. S. Lang, W. Riedmoeller, and M. Salvat, Appl. Phys. Lett. 30, 461 (1977).

[5] W. Yu, H. Xu, F. He, M. Y. Yu, S. Ishiguro, J. Zhang, and A. Y. Wong, Phys. Rev. E 72, 046401 (2005).

[6] S. S. Bulanov, A. Brantov, V. Y. Bychenkov, V. Chvykov, G. Kalinchenko, T. Matsuoka, P. Rousseau, S. Reed, V. Yanovsky, D. W. Litzenberg, K. Krushelnick, and A. Maksimchuk, Phys. Rev. E 78, 026412 (2008).

[7] T. Kluge, W. Enghardt, S. D. Kraft, U. Schramm, K. Zeil, T. E. Cowan, and M. Bussmann, Phys. Plasmas 17, 123103 (2010).

[8] J. Limpouch, J. Psikal, A. Andreev, K. Y. Platonov, and S. Kawata, Laser Part. Beams 26, 225 (2008).

[9] F. Peano, R. A. Fonseca, and L. O. Silva, Phys. Rev. Lett. 94, 033401 (2005).

[10] F. Peano, F. Peinetti, R. Mulas, G. Coppa, and L. O. Silva, Phys. Rev. Lett. 96, 175002 (2006).

[11] M. Murakami and M. Tanaka, Phys. Plasmas 15, 082702 (2008).

[12] T. Sokollik, M. Schnürer, S. Steinke, P. V. Nickles, W. Sandner, M. Amin, T. Toncian, O. Willi, and A. A. Andreev, Phys. Rev. Lett. 103, 135003 (2009).

[13] S. Karsch, S. Düsterer, H. Schwoerer, F. Ewald, D. Habs, M. Hegelich, G. Pretzler, A. Pukhov, K. Witte, and R. Sauerbrey, Phys. Rev. Lett. 91, 015001 (2003).

[14] S. Ter-Avetisyan, M. Schnürer, P. V. Nickles, M. Kalashnikov, E. Risse, T. Sokollik, W. Sandner, A. Andreev, and V. Tikhonchuk, Phys. Rev. Lett. 96, 145006 (2006).

[15] S. Ter-Avetisyan, B. Ramakrishna, R. Prasad, M. Borghesi, P. V. Nickles, S. Steinke, M. Schnürer, K. I. Popov, L. Ramunno, N. V. Zmitrenko, and V. Yu. Bychenkov, Phys. Plasmas 19, 073112 (2012).
[16] T. Ditmire, J. Zweiback, V. Yanovsky, T. Cowan, G. Hays, and K. Wharton, Nature (London) 398, 489 (1999).

[17] Y. Fukuda, A. Y. Faenov, M. Tampo, T. A. Pikuz, T. Nakamura, M. Kando, Y. Hayashi, A. Yogo, H. Sakaki, T. Kameshima, A. S. Pirozhkov, K. Ogura, M. Mori, T. Z. Esirkepov, J. Koga, A. S. Boldarev, V. A. Gasilov, A. I. Magunov, T. Yamauchi, R. Kodama, P. R. Bolton, Y. Kato, T. Tajima, H. Daido, and S. V. Bulanov, Phys. Rev. Lett. 103, 165002 (2009).

[18] A. Henig, D. Kiefer, M. Geissler, S. G. Rykovanov, R. Ramis, R. Hörlein, J. Osterhoff, Z. Major, L. Veisz, S. Karsch, F. Krausz, D. Habs, and J. Schreiber, Phys. Rev. Lett. 102, 095002 (2009).

[19] K. Zeil, J. Metzkes, T. Kluge, M. Bussmann, T. Cowan, S. Kraft, R. Sauerbrey, B. Schmidt, M. Zier, and U. Schramm, Plasma Phys. Control. Fusion 56, 084004 (2014).

[20] T. Sokollik, T. Paasch-Colberg, K. Gorling, U. Eichmann, M. Schnürer, S. Steinke, P. V. Nickles, A. Andreev, and W. Sandner, New J. Phys. 12, 113013 (2010).

[21] W. Paul, Angew. Chem. Int. Ed. Engl. 29, 739 (1990).

[22] Tectra GmbH, www.tectra.de.

[23] Microparticles GmbH, Berlin, http://www.microparticles.com.

[24] D. Jung, R. Hörlein, D. C. Gautier, S. Letzring, D. Kiefer, K. Allinger, B. J. Albright, R. Shah, S. Palaniyappan, L. Yin, J. C. Fernández, D. Habs, and B. M. Hegelich, Rev. Sci. Instrum. 82, 043301 (2011)

[25] M. Murakami, Y.-G. Kang, K. Nishihara, S. Fujioka, and H. Nishimura, Phys. Plasmas 12, 062706 (2005).

[26] V. Kovalev, V. Y. Bychenkov, and V. Tikhonchuk, J. Exp. Theor. Phys. 95, 226 (2002).

[27] S. C. Wilks, W. L. Kruer, M. Tabak, and A. B. Langdon, Phys. Rev. Lett. 69, 1383 (1992).

[28] T. Kluge, T. Cowan, A. Debus, U. Schramm, K. Zeil, and M. Bussmann, Phys. Rev. Lett. 107, 205003 (2011).

[29] Y. Ping, R. Shepherd, B. F. Lasinski, M. Tabak, H. Chen, H. K. Chung, K. B. Fournier, S. B. Hansen, A. Kemp, D. A. Liedahl, K. Widmann, S. C. Wilks, 
W. Rozmus, and M. Sherlock, Phys. Rev. Lett. 100, 085004 (2008).

[30] S. Sakabe, S. Shimizu, M. Hashida, S. Sato, T. Tsuyukushi, K. Nishihara, S. Okihara, T. Kagawa, Y. Izawa, K. Imasaki, and T. Iida, Phys. Rev. A 69, 023203 (2004).

[31] A. V. Brantov, V. T. Tikhonchuk, O. Klimo, D. V. Romanov, S. Ter-Avetisyan, M. Schnürer, T. Sokollik, and P. V. Nickles, Phys. Plasmas 13, 122705 (2006).
[32] H. Ruhl, K. Bamberg, F. Deutschmann, C. Klier, N. Moschüring, and K. Germaschewski, Plasma Simulation Code, http://www.plasma-simulation-code.net, http://fishercat.sr.unh.edu/psc/index.html.

[33] A. J. Kemp and H. Ruhl, Phys. Plasmas 12, 033105 (2005).

[34] M. Schnürer, S. Ter-Avetisyan, S. Busch, E. Risse, M. P. Kalachnikov, W. Sandner, and P. V. Nickles, Laser Part. Beams 23, 337 (2005). 